\title{
Novel Composite Approximation for the Gaussian Q-Function
}

\author{
Zoran H. Peric ${ }^{1}$, Aleksandar V. Markovic ${ }^{2,}$, Natasa Z. Kontrec ${ }^{2}$, Stefan R. Panic ${ }^{2}$, Petar C. Spalevic ${ }^{3}$ \\ ${ }^{1}$ Faculty of Electronic Engineering, University of Nis, \\ Aleksandra Medvedeva 14, 18000 Nis, Serbia \\ ${ }^{2}$ Faculty of Science, University of Pristina - Kosovska Mitrovica, \\ Lole Ribara 29, 38220 Kosovska Mitrovica, Serbia \\ ${ }^{3}$ Faculty of Technical Sciences, University of Pristina, \\ Kosovska Mitrovica, Serbia \\ aleksandar.markovic@pr.ac.rs
}

\begin{abstract}
This paper, by using Borjesson's and Benitez's approximation of $Q$-function, presents a novel and improved composite approximation of $Q$-function with wide applicability. The presented approach is very general and can be implemented on any observed interval. Based on the proposed approximation of $Q$-function, the average bit error rate is assessed by observing the transfer over Nakagami-m fading channel. The simplicity of the proposed approximation form in conjunction with yet another feature - utmost accurateness appeared to be a better choice than the suggested approximations of similar complexity in terms of analyticity. The paper emphasizes the wide implementation possibilities in numerous tasks of communication theory and functional analysis that include $Q$-function.
\end{abstract}

Index Terms-Gaussian distribution; Function approximation; Modulation; Nakagami distribution.

\section{INTRODUCTION}

The wireless communication systems' advancement is followed by the urge to construct methods and models for implementation of related special functions' approximations to achieve an efficient assessment of related performances of these systems. To evaluate the behavior of digital communication systems, it is crucial to set standard performance baseline measures for the examined digital communication systems for the implementation of various types of modulation, detection types, channel models, and also it is necessary to examine the dependence of performance on the key system parameter values. The value of the Average Symbol Error Rate (ASER) at the reception for the implemented modulation scheme is a performance measure that best describes the nature of the communication system's behavior. To analytically assess ASER for the implemented modulation format, it is of great importance to apply the most precise approximation of the special functions, such as $\operatorname{erfc}(x), \operatorname{erf}(x), \operatorname{Marcum} Q$, and $Q$-function, for the widest input range values. This problem has already been researched in literature [1]-[10], but the pursuit for a

Manuscript received 28 February, 2020; accepted 25 July, 2020

This work is supported by Serbian Ministry of Education and Science under Grants No. TR35030, TR32023, and TR35026. simpler and more accurate approximation of $Q$-function is still ongoing due to the continual development of different wireless services.

This paper presents the composite method for the approximation of the $Q$-function.

Along with straightforwardness regarding the realization of the examined functions' approximations, yet another characteristic is examined - the robustness of approximations of both absolute and relative error rates with regard to a broad scope of values of entry parameters.

This solution has clear advantages, which are stressed in a direct comparison with both absolute and relative errors attained by using the known approximations of $Q$-function from related sources found in literature. Moreover, during the data transmission over fading communication channels, for cases where the proposed $Q$-function approximation is implemented, it is verified that that system performances can be assessed with far greater ease by utilizing the solutions proposed in the paper. Consequently, it is also easier to determine the communication Symbol Error Rate (SER) over various communication channel conditions. Further, comparing the foreseen values of the ASER at reception, during the signal transmission through different media of communication channels, for cases where the existing, previously proposed approximations of $Q$-function are implemented, also attests to the fact that the communication system's performances can be calculated more accurately. As a result, a novel, simpler, and highly accurate approximation of $Q$-function is obtained, and it will be further used for SER transmission estimation over the Nakagami- $m$ channel.

This approximation of Gaussian $Q$-function is based on characteristics of Borjesson's approximation [2] and Benitez's exponential approximation [11] for $Q$-function, but taking into account the composite characteristics of minimization Mean Square Error (MSE).

A comparison with other known $Q$-function approximations is conducted and the results are presented in Section IV ("Numerical results"). Also, it is shown that by using the proposed approximation, the Average Symbol Error Rate (ASER) values for Binary Phase-Shift Keying 
(BPSK) and Differentially Encoded-Quadrature Phase Shift Keying (DE-QPSK) implemented modulation format can be efficiently and accurately assessed when the transfer is observed over Nakagami- $m$ channel.

\section{Q-FUnCTION APPROXIMATION ACCURACY ANALYSIS}

Gaussian $Q$-function $Q(x)$ has a major role in performance analysis of different communication schemes where the noise is represented as a Gaussian random variable [12]. In particular, the problem comes down to finding the closedform formula that approximates $Q$-function given with (1). $Q$-function is defined as a complementary function of the cumulative distribution of Gaussian random variable $X$ with zero mean and unit variance [1]-[11]

$$
\begin{gathered}
Q(x)=\frac{1}{\sqrt{2 \pi}} \int_{x}^{\infty} \exp \left\{-\frac{t^{2}}{2}\right\} d t=\frac{1}{2} \operatorname{erfc}\left(\frac{x}{\sqrt{2}}\right)= \\
=\frac{1}{2}\left[1-\operatorname{erf}\left(\frac{x}{\sqrt{2}}\right)\right] .
\end{gathered}
$$

Two particularly important functions are error function, $\operatorname{erf}(x)$, and complementary error function, $\operatorname{erfc}(x)$, defined as follows:

$$
\begin{gathered}
\operatorname{erf}(x)=\frac{2}{\sqrt{\pi}} \int_{0}^{x} \exp \left\{-t^{2}\right\} d t \\
\operatorname{erfc}(x)=1-\operatorname{erf}(x)=\frac{2}{\sqrt{\pi}} \int_{x}^{+\infty} \exp \left\{-t^{2}\right\} d t .
\end{gathered}
$$

Borjesson and Sundberg [2] proposed the definite integral and solid approximation of function $Q(x)$ stressing the simplicity and applicability of their proposal

$$
\begin{gathered}
Q_{a-\text { Borjesson-1 }}(x)= \\
=\frac{1}{\sqrt{2 \pi}} \frac{1}{(1-a) x+a \sqrt{x^{2}+b}} \exp \left\{-\frac{x^{2}}{2}\right\},
\end{gathered}
$$

where $a$ and $b$ are scalar fitting parameters having optimal values that amount to $a=0.339$ and $b=5.510$.

Chiani, Dardari, and Simon presented approximation of the $Q$-function in a form of the sum of exponential functions [3]. This approximation has a relatively simple analytical form, but is less accurate

$$
Q_{\text {Chiani }}(x)=\frac{1}{12} \exp \left\{-\frac{x^{2}}{2}\right\}+\frac{1}{4} \exp \left\{-\frac{2 x^{2}}{3}\right\} .
$$

Karagiannidis and Lioumpas [5] proposed a new, simple, and tight approximation for the Gaussian $Q$-function which proved highly accurate for smaller values of arguments

$$
\begin{gathered}
Q_{a-\text { Karagiannidis }}(x)= \\
=\frac{1}{\sqrt{2 \pi}} \frac{\left(1-\exp \left\{-A \frac{x}{\sqrt{2}}\right\}\right)}{B x} \exp \left\{-\frac{x^{2}}{2}\right\},
\end{gathered}
$$

where values of $A$ and $B$ were found numerically as 1.98 and 1.135, respectively, for the argument region $(x \in[0,20])$.

The Prony (sum-of-exponentials) approximation of $Q$ function proposed by Loskot and Beaulieu [13] in terms of two exponential functions is given with

$$
\begin{gathered}
Q_{a-\text { Loskot } 1}(x)=0.208 \exp \left\{-0.971 x^{2}\right\}+ \\
+0.147 \exp \left\{-0.525 x^{2}\right\},
\end{gathered}
$$

and in terms of three exponential function, it is

$$
\begin{gathered}
Q_{a-\text { Loskot } 2}(x)=0.168 \exp \left\{-0.876 x^{2}\right\}+ \\
+0.144 \exp \left\{-0.525 x^{2}\right\}+0.002 \exp \left\{-0.603 x^{2}\right\} .
\end{gathered}
$$

To further improve the accuracy of the Gaussian $Q$ function with a minor increase of the computational complexity, Develi [14] proposed a new approximation

$$
\begin{gathered}
Q_{a-\text { Develi }}(x)= \\
=\frac{1}{\sqrt{2 \pi}} \frac{0.9702 \times \exp \left\{-\frac{x^{2}}{2.0055}\right\}}{\left(0.3987 \sqrt{x^{2}+3.6677}+(1-0.3987) x\right)}- \\
-\left(0.0013 \exp \left\{-2.369 x^{2}\right\}+0.0054 \exp \left\{-1.0436 x^{2}\right\}\right),
\end{gathered}
$$

which can make the exact prediction of the Gaussian $Q$ function, in particular for the small values of argument $x$. However, it should be noted that its exactness can have a proportionally decreasing tendency as the argument $x$ 's values rise up. In [15], another approximation of the Gaussian $Q$-function with two exponential terms was proposed, denoted as $Q_{a \text {-Sofotasios }}(x)$

$$
Q_{a-\text { Sofotasios }}(x)=0.49 \exp \left\{-\frac{8}{13} x\right\} \exp \left\{-\frac{x^{2}}{2}\right\} \text {. }
$$

The second-order exponential function presented in [11], denoted as $Q_{a-B e n i t e z}(x)(11)$, with minimum square sum error (min-SSE), has significant approximate errors beside for the range $x \in[0,2]$. Hence, this approximation is less convenient for use when the performance of the communication system under the influence of fading is calculated

$$
Q_{a-\text { Benitez }}(x)=\exp \left\{a x^{2}+b x+c\right\} \text {. }
$$

\section{MAIN RESUlt}

The most significant attainment of this paper is the new composite $Q$-function approximation, which is elaborated in particular in the forthcoming part.

In search of the simplest approximation with high accuracy, the approximation of $Q$-function proposed in [16] can be used as a starting point 


$$
Q_{m}(x)=\exp \left\{a_{n} x^{n}+a_{n-1} x^{n-1}+\cdots+a_{1} x+a_{0}\right\},
$$

where $n$ denotes the approximation order and $\Omega=\left[a_{n}, a_{n-1}, \ldots, a_{0}\right]$ unknown parameters. Equation (12) can be presented in a closed-form as in [16]

$$
Q_{m}(x)=f\left(x, a_{n}, a_{n-1}, \ldots, a_{0}\right),
$$

where $f(x)$ denotes non-linear correlation between $\left\{x, a_{n}, a_{n-}\right.$ $\left.1, \ldots, a_{0}\right\}$ and $Q_{m}(x)$. For a considered $n$, values of $\Omega$ are calculated with MSE minimization in order to achieve the best approximation of $Q$-function for the observed range $x$

$$
Q_{M S E}(x ; \Omega)=\frac{1}{N} \sum_{k=1}^{N}\left(Q\left(x_{k}\right)-Q_{m}\left(x_{k}\right)\right)^{2},
$$

where $k$ denotes $k^{\text {th }}$ sample, $N$ denotes the number of samples. $Q\left(x_{k}\right)$ denotes accurate values of $Q$-function, while $Q_{m}\left(x_{k}\right)$ denotes values calculated with the proposed approximation. Equation (14) can be stated as

$$
\begin{gathered}
Q_{M S E}(x ; \Omega)=\frac{1}{N} \int_{k=0}^{N}\left(Q\left(x_{k}\right)-\exp \left\{a_{n} x^{n}+a_{n-1} x^{n-1}+\cdots+a_{1} x+a_{0}\right\}\right) d x \approx \\
\approx \frac{1}{N} \sum_{k=0}^{N}\left(Q\left(x_{k}\right)-Q_{\left(a_{n}, a_{n-1}, \ldots, a_{0}\right)}\left(x_{k}\right)\right)^{2} .
\end{gathered}
$$

This new approximation of $Q$-function, as a result of MSE application, can be defined as:

$$
\begin{gathered}
Q_{M S E}(x)= \begin{cases}Q_{1}(x), & 0 \leq x \leq x_{1}, \\
Q_{2}(x), & x>x_{1},\end{cases} \\
Q_{1}(x)=\exp \left\{a_{0} x^{2}+a_{1} x+a_{2}\right\}, \\
Q_{2}(x)=\frac{1}{\sqrt{2 \pi}} \frac{1}{\left(1-b_{1}\right) x+b_{1} \sqrt{x^{2}+c_{1}}} \exp \left\{-\frac{x^{2}}{2}\right\} .
\end{gathered}
$$

Examining a characteristic that accuracy in approximating the $Q$-function is dependable on the fitting parameters resulted in the idea to construct this particular composite function, which is also highly dependable on the argument's range.

The use of MSE for the observed ranges $x \in\left[0, x_{1}\right]$ and $x \in\left[x_{1}, \infty\right]$ resulted in the related values for parameters $x_{l}$, $a_{0}, a_{1}, a_{2}, b_{1}$, and $c_{1}$. For $x_{1}=0.7$, the values are $a_{0}=-$ $0.35054, a_{1}=-0.78995, a_{2}=-0.69354$, and $b_{1}=0.32026$, and $c_{1}=5.97353$

$$
\left[\begin{array}{c}
Q_{M S E}(x)=\left\{\begin{array}{lc}
Q_{1}(x), & 0 \leq x \leq 0.7 \\
Q_{2}(x), & x>0.7
\end{array}\right. \\
Q_{1}(x)=\exp \left\{-0.35054 x^{2}-0.78995 x-0.69354\right\} \\
Q_{2}(x)=\frac{1}{\sqrt{2 \pi}} \frac{\exp \left\{-\frac{x^{2}}{2}\right\}}{0.6797 x+0.3202 \sqrt{x^{2}+5.9735}} .
\end{array}\right.
$$

In order to optimize $Q$-function approximation in terms of observed parameter range, we provided two forms of $Q_{\mathrm{MSE}}$ approximation presented with (16). The first approximation form $Q_{1}(x)$ yields better results for the small range of parameter $x(0 \leq x \leq 0.7)$, while for the higher range of this parameter ( $x>0.7), Q_{2}(x)$ approximation is more suitable. It can be noticed that the better $Q$-function approximation for the small range of parameter $x$ has been obtained with the second-order approximation, so there was no need for further consideration.

\section{NUMERICAL RESULTS}

In this section, we are comparing the accuracy of the proposed approximation of the $Q$-function with the already existing approximations of the $Q$-function available in the literature. In the literature, different empirical and analytical methods of approximation have been presented, providing different compromises between the accuracy of the $Q$ function approximation and the analytical complexity. Also, some of the available approximations, despite adequate analytical complexity, continue to provide insufficient accuracy. Particular methods can prove appropriate for small arguments, but not for large arguments and likewise. In other words, in this section, we will show the validity of the proposed composite approximation of the $Q$-function by comparing its characteristics with the already existing approximations of the $Q$-function.

Table I exemplifies the comparison or $Q$-function and its approximations. Evidently, the improvement in accuracy is achieved throughout the range of values by using this new proposed approximation (17) with the assistance of MSE.

In order to compare as described in numerous papers on the related subject, we have decided to select a common interval of argument $x$ ranging $[0,6]$, since we have determined the dependences of the absolute relative errors of approximating the $Q$-function on the argument $x$, as presented in Fig. 1.

After the evaluation of optimization parameters, it has been examined how the proposed approximation is achieved with the use of MSE denotes Gaussian $Q$-function. In Fig. 2, the proposed approximation is compared with the approximations proposed by Karagiannidis and Borjesson, observed through the prism of the absolute relative error values as a function of argument $x$. 
TABLE I. COMPARISON OF ERF(X) FUNCTION APPROXIMATIONS.

\begin{tabular}{|c|c|c|c|c|c|}
\hline $\mathbf{x}$ & $\mathrm{x}=0.1$ & $\mathrm{x}=0.3$ & $x=0.5$ & $x=0.7$ & $x=1$ \\
\hline $\operatorname{erf}(x)$ & 0.539828 & 0.617911 & 0.691462 & 0.758036 & 0.841345 \\
\hline $\operatorname{erf}_{\text {MSE }}(x)$ & 0.539805 & 0.6179 & 0.691536 & 0.758467 & 0.841379 \\
\hline $\operatorname{erf}_{\text {Bor.-1 }}(x)$ & 0.539802 & 0.618811 & 0.692277 & 0.758519 & 0.841429 \\
\hline $\operatorname{erf}_{\text {Kar. }}(x)$ & 0.543074 & 0.615849 & 0.687681 & 0.754478 & 0.839378 \\
\hline $\operatorname{erf}_{\text {Chian }}(x)$ & 0.668743 & 0.684892 & 0.714838 & 0.754444 & 0.821101 \\
\hline $\operatorname{erf}_{\text {Losk. }}(\mathbf{x})$ & 0.64778 & 0.66919 & 0.707912 & 0.757093 & 0.834271 \\
\hline $\operatorname{erf}_{\text {Sofot. }}(\mathbf{x})$ & 0.541543 & 0.610529 & 0.682108 & 0.750705 & 0.839383 \\
\hline $\operatorname{erf}_{\text {Benit. }}(x)$ & 0.541574 & 0.616848 & 0.68954 & 0.756121 & 0.839795 \\
\hline $\mathbf{x}$ & $x=2$ & $\mathbf{x}=\mathbf{3}$ & $x=4$ & \multicolumn{2}{|c|}{$x=5$} \\
\hline $\operatorname{erf}(x)$ & 0.97725 & 0.99865 & 0.999968 & \multicolumn{2}{|c|}{1} \\
\hline $\operatorname{erf}_{\mathrm{MSE}}(\mathbf{x})$ & 0.977228 & 0.998648 & 0.999968 & \multicolumn{2}{|c|}{1} \\
\hline $\operatorname{erf}_{\text {Bor.-1 }}(x)$ & 0.977194 & 0.998646 & 0.999967 & \multicolumn{2}{|c|}{1} \\
\hline $\operatorname{erf}_{\text {Kar. }}(x)$ & 0.977662 & 0.998718 & 0.999971 & \multicolumn{2}{|c|}{1} \\
\hline $\operatorname{erf}$ Chian $(x)$ & 0.971351 & 0.998455 & 0.999966 & \multicolumn{2}{|c|}{1} \\
\hline $\operatorname{erf}_{\text {Losk. }}(x)$ & 0.977721 & 0.998663 & 0.999967 & \multicolumn{2}{|c|}{1} \\
\hline $\operatorname{erf}_{\text {Sofot. }}(x)$ & 0.980632 & 0.999141 & 0.999986 & \multicolumn{2}{|c|}{1} \\
\hline $\operatorname{erf}_{\text {Benit. }}(\mathbf{x})$ & 0.976148 & 0.998364 & 0.999948 & \multicolumn{2}{|c|}{0.999999} \\
\hline
\end{tabular}

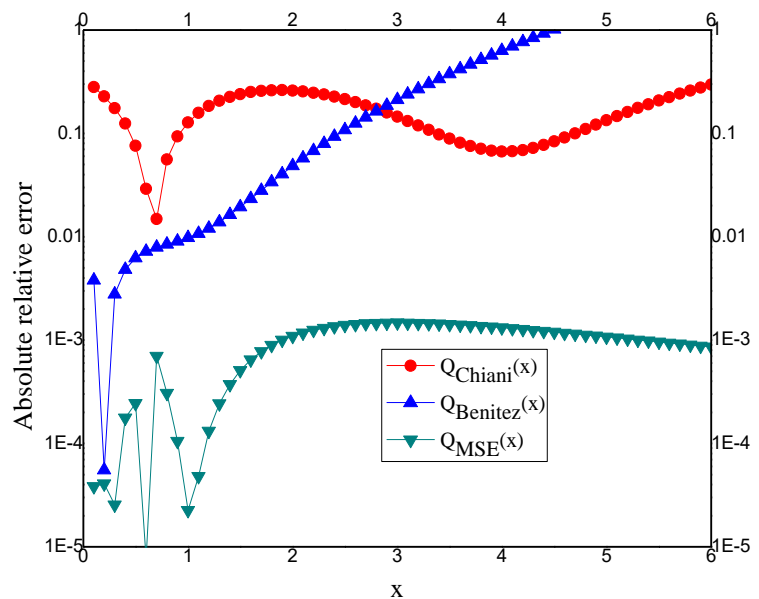

Fig. 1. Comparison between values of absolute relative errors of Chiani, Benitez approximations and the proposed composite method of approximation.

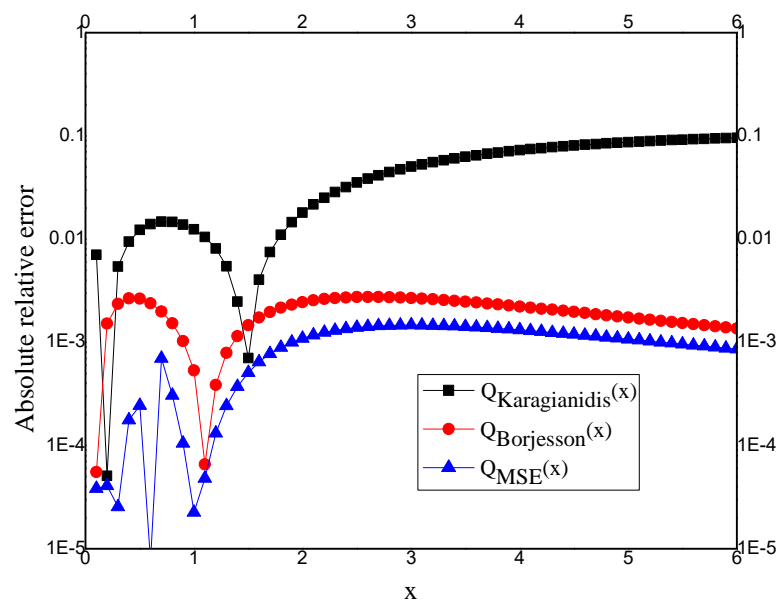

Fig. 2. Comparison between values of absolute relative errors of Karagiannidis, Borjesson approximations and the proposed composite method of approximation.

As depicted in Fig. 2, all previously proposed approximations have low accuracy compared to the proposed approximation. The proposed approximation offers a significant improvement in accuracy compared to Karagiannidis and Borjesson proposed approximations throughout the range, and particularly for smaller values of argument $x$.

Figure 3 and Figure 4 show that the proposed method has the lowest values of the absolute relative error, i.e., improvement in accuracy has been achieved throughout the range.

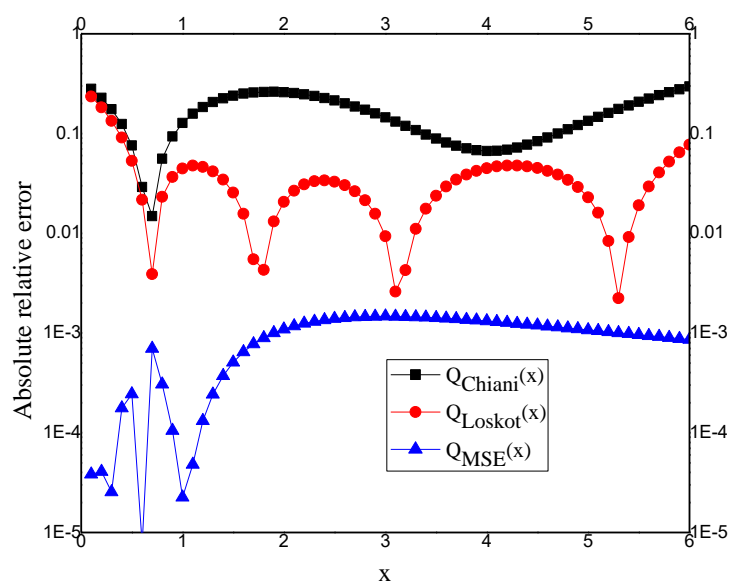

Fig. 3. Comparison between values of absolute relative errors of Chiani, Loskot approximations with the proposed composite method of approximation.

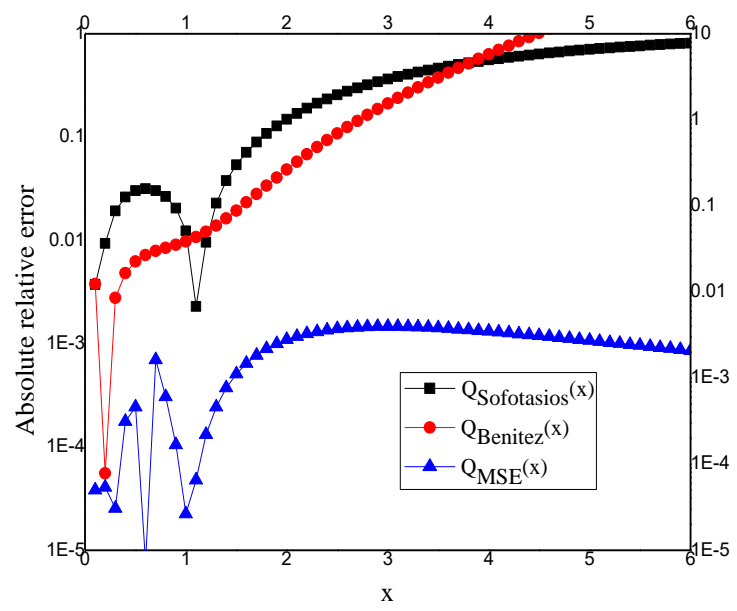

Fig. 4. Comparison between values of absolute relative errors of Sofotasios, Benitez approximations with the proposed composite method of approximation.

Figure 5 provides ASER values for the BPSK format of 
modulation in the presence of the Nakagami- $m$ fading channel. To highlight the excellent match of Average Symbol Error Probability (ASEP) values for BPSK format modulation accomplished by using the proposed composite method with accurate values, Fig. 6 provides values for the absolute relative error of ASEP for BPSK proposed approximations for different values of Nakagami- $m$ fading channel. Resulting from the use of the proposed method, highly accurate $Q$-function approximations (low values of absolute relative error) in a wide range of input arguments have been achieved compared to other known approximations for calculating ASEP values.

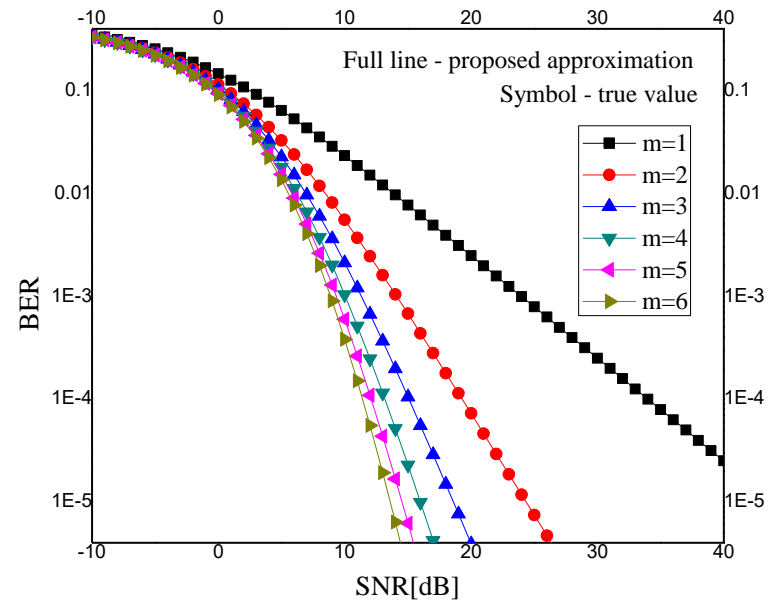

Fig. 5. Accurate and approximate ASER values for BPSK.

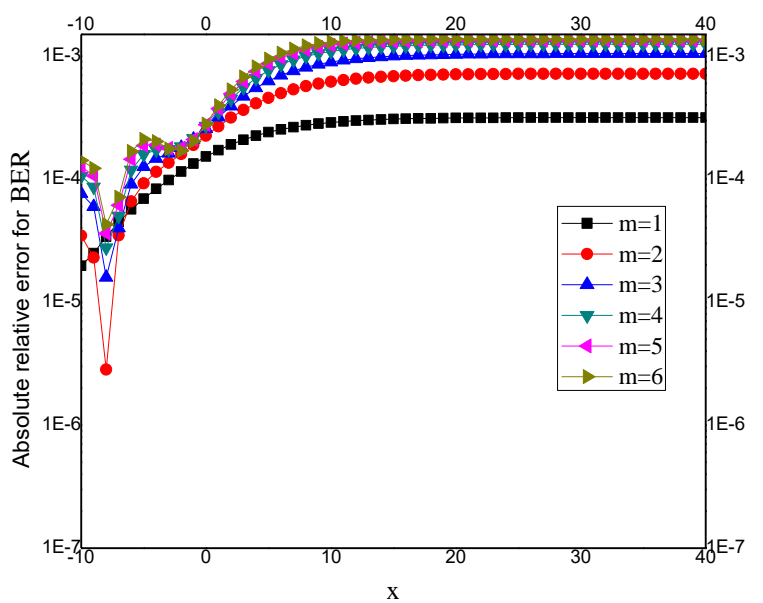

Fig. 6. The absolute relative error for ASER for BPSK.

Implementation of the composite method of $Q$-function approximation can also be extended to the calculation of the average error rate for DE-QPSK. Figure 7 provides ASER values for DE-QPSK modulation format in the presence of the Nakagami- $m$ fading channel. Figure 8 provides values of the absolute relative error of the proposed approximation ASER for DE-QPSK.

Based on Fig. 7 and Fig. 8, it can be concluded that ASEP values for DE-QPSK implemented modulation format in the presence of the Nakagami- $m$ fading channel for different values of parameter $m$ can be efficiently and accurately evaluated with the use of the proposed composite method. By using the proposed approximation, the performance measures for ASER have been evaluated more accurately than by using any other known $Q$-function approximations in closed-form throughout the range of input values.

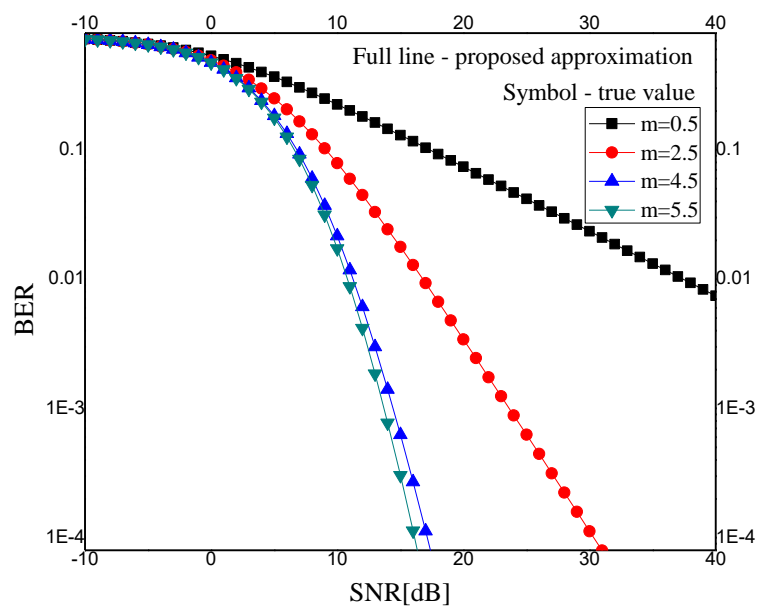

Fig. 7. Accurate and approximate ASER values for DE-QPSK.

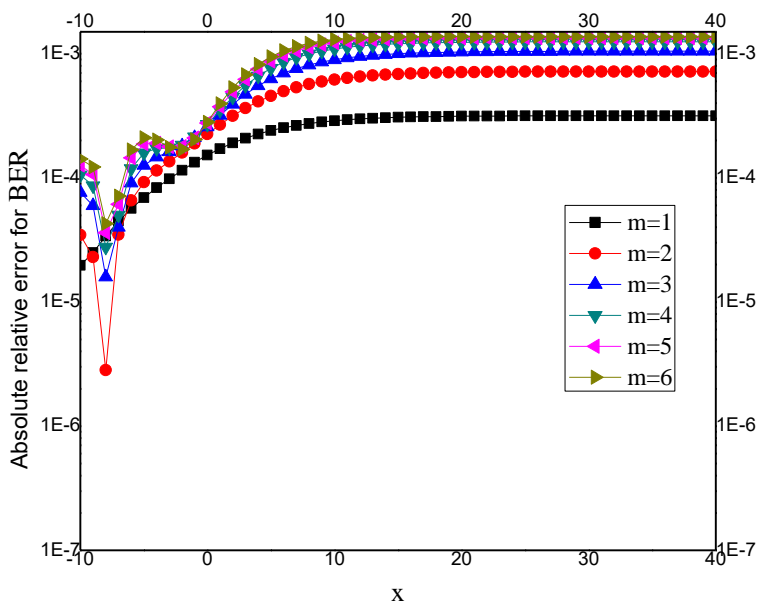

Fig. 8. The absolute relative error of the proposed approximation ASEP for DE-QPSK.

\section{CONCLUSIONS}

This paper provides a novel and improved composite $Q$ function approximation. The comparison with other known closed-form $Q$-function approximations, which has been provided at Table $I$ and at Figs. 1-4, verifies the improvement in accuracy throughout the domain of function argument values. Moreover, the comparison between ASEP values has been provided and presented at Figs. 5-8. The comparison has been presented by the use of a novel and improved composite $Q$-function approximation, along with other well-known $Q$-function approximations, for cases when BPSK and DE-QPSK are being observed. The proposed approximation can also be useful in tackling numerous problems in communication theory.

\section{CONFLICTS OF INTEREST}

The authors declare that they have no conflicts of interest.

\section{REFERENCES}

[1] C. Tellambura and A. Annamalai, "Efficient computation of erfc(x) for large arguments", IEEE Transactions on Communications, vol. 48, no. 4, pp. 529-532, Apr. 2000. DOI: 10.1109/26.843116.

[2] P. O. Borjesson and C.-E. Sundberg, "Simple approximations of the error function $\mathrm{Q}(\mathrm{x})$ for communications applications", IEEE Transactions on Communications, vol. 27, no. 3, pp. 639-643, Mar. 
1979. DOI: 10.1109/TCOM.1979.1094433.

[3] M. Chiani, D. Dardari, and M. K. Simon, "New exponential bounds and approximations for the computation of error probability in fading channels", IEEE Trans. Wireless Commun., vol. 2, no. 4, pp. 840845, Jul. 2003. DOI: 10.1109/TWC.2003.814350.

[4] A. Markovic, Z. Peric, S. Panic, P. Spalevic, and Z. Todorovic, "Improved composite Q-function approximation and its application in ASEP of digital modulations over fading channels", Elektronika ir Elektrotechnika, vol. 23, no. 3, pp. 83-88, 2017. DOI: 10.5755/j01.eie.23.3.18338.

[5] G. K. Karagiannidis and A. S. Lioumpas, "An improved approximation for the Gaussian Q-function", IEEE Commun. Lett., vol. 11, no. 8, pp. 644-646, Aug. 2007. DOI: 10.1109/LCOMM.2007.070470.

[6] A. V. Markovic, Z. H. Peric, S. R. Panic, P. C. Spalevic, and B. P. Prlincevic, "An improved method for ASEP evaluation over fading channels based on Q function approximation", IETE Journal of Research, vol. 64, no. 6, pp. 777-784, Nov.-Dec. 2018. DOI 10.1080/03772063.2017.1369910.

[7] W. M. Jang, "A simple upper bound of the Gaussian Q-function with closed-form error bound", IEEE Commun. Lett., vol. 15, no. 2, pp. 157-159, Feb. 2011. DOI: 10.1109/LCOMM.2011.011011.102207.

[8] P. Fan, "New inequalities of Mill's ratio and application to the inverse Q-function approximation", Australian J. Math. Anal. Appl., vol. 10, no. 1, pp. 1-11, 2013.

[9] Q. Shi and Y. Karasawa, "An accurate and efficient approximation to the Gaussian Q-Function and its applications in performance analysis in Nakagami-m fading", IEEE Commun. Lett., vol. 15, no. 5, pp.
479-481, May 2011. DOI: 10.1109/LCOMM.2011.032111.102440.

[10] J. Nikolic, Z. Peric, and Aleksandar Markovic, "Proposal of simple and accurate two-parametric approximation for the Q-function", Mathematical Problems in Engineering, vol. 2017, article ID 8140487. DOI: $10.1155 / 2017 / 8140487$.

[11] M. Lopez-Benitez and F. Casadevall, "Versatile, accurate, and analytically tractable approximation for the Gaussian Q-function", IEEE Trans. Commun., vol. 59, no. 4, pp. 917-922, 2011. DOI 10.1109/TCOMM.2011.012711.100105.

[12] M. K. Simon, Probability Distributions Involving Gaussian Random Variables: A Handbook for Engineers and Scientists. Boston, MA: Kluwer, 2002.

[13] P. Loskot and N. C. Beaulieu, "Prony and polynomial approximations for evaluation of the average probability of error over slow-fading channels", IEEE Transactions on Vehicular Technology, vol. 58, no. 3, pp. 1269-1280, 2009. DOI: 10.1109/TVT.2008.926072.

[14] I. Develi, "A new approximation based on the differential evolution algorithm for the Gaussian Q-function", International Journal Innovative Computing Information and Control, vol. 8, no. 10B, pp. 7095-7102, 2012

[15] P. C. Sofotasios and S. Freear, "Novel expressions for the Marcum and one dimensional Q-functions", in Proc. of 2010 7th International Symposium on Wireless Communication Systems, 2010, pp. 736-740. DOI: 10.1109/ISWCS.2010.5624374.

[16] I. Develi and A. Akdagli, "High-order exponential approximations for the Gaussian Q-function obtained by genetic algorithm", International Journal of Electronics, vol. 100, no. 4, pp. 553-562, 2013. DOI: $10.1080 / 00207217.2012 .713024$

This article is an open access article distributed under the terms and conditions of the Creative Commons Attribution 4.0 (CC BY 4.0) license (http://creativecommons.org/licenses/by/4.0/). 